\title{
Drug-Drug Interaction Extraction via Convolutional Neural Networks
}

\author{
Shengyu Liu, Buzhou Tang, Qingcai Chen, and Xiaolong Wang \\ Key Laboratory of Network Oriented Intelligent Computation, Harbin Institute of Technology Shenzhen Graduate School,
} Shenzhen 518055, China

Correspondence should be addressed to Qingcai Chen; qingcai.chen@gmail.com

Received 11 October 2015; Accepted 21 December 2015

Academic Editor: Liangjiang Wang

Copyright (C) 2016 Shengyu Liu et al. This is an open access article distributed under the Creative Commons Attribution License, which permits unrestricted use, distribution, and reproduction in any medium, provided the original work is properly cited.

\begin{abstract}
Drug-drug interaction (DDI) extraction as a typical relation extraction task in natural language processing (NLP) has always attracted great attention. Most state-of-the-art DDI extraction systems are based on support vector machines (SVM) with a large number of manually defined features. Recently, convolutional neural networks $(\mathrm{CNN})$, a robust machine learning method which almost does not need manually defined features, has exhibited great potential for many NLP tasks. It is worth employing CNN for DDI extraction, which has never been investigated. We proposed a CNN-based method for DDI extraction. Experiments conducted on the 2013 DDIExtraction challenge corpus demonstrate that CNN is a good choice for DDI extraction. The CNN-based DDI extraction method achieves an $F$-score of $69.75 \%$, which outperforms the existing best performing method by $2.75 \%$.
\end{abstract}

\section{Introduction}

Drug-drug interactions (DDIs) occur when two or more drugs are taken in combination that alters the way one or more drugs act in human body and may result in unexpected side effects. The unexpected side effects caused by DDIs are always very dangerous (may lead to deaths) and greatly increase healthcare costs. The more DDIs healthcare professionals know, the less medical accidents occur. Therefore, DDIs have always been attracting much attention in drug safety and healthcare management [1]. There are several publicly available databases supporting healthcare professionals to find DDIs. For example, DrugBank [2], which is an online drug database, consists of 8311 drugs entries. Each drug entry contains more than 200 fields, including a DDI field. However, the databases have a few limitations. Firstly, most DDI databases are dictionaries with a DDI field described in text such as DrugBank. The DDIs in these databases cannot be directly accessed like relational databases by healthcare professionals. Secondly, new DDIs are often detected by healthcare professionals and presented in literature, including scientific articles, books, and technical reports [3]. It is impossible for healthcare professionals to find DDIs from the overwhelming amount of literature manually and to keep up-to-date with the latest DDI findings. Therefore, DDI extraction, which detects DDIs in unstructured text and classifies them into predefined categories automatically, has become an increasing interest in medical text mining.

DDI extraction is a typical relation extraction task in natural language processing (NLP). Many methods have been proposed for DDI extraction and can be divided into two categories: rule-based [4] and machine learning-based methods $[5,6]$. Rule-based methods use manually defined rules to extract DDIs, whereas machine learning-based methods treat DDI extraction as a standard supervised learning problem over annotated corpora. Compared with rule-based methods, machine learning-based methods usually show better performance and better portability [7]. Due to lack of annotated corpora, early DDI extraction methods are almost all rulebased. For example, Segura-Bedmar et al. [4] defined a set of domain-specific rules to extract DDIs in DrugBank. With the organization of DDIExtraction challenges in 2011 and 2013 [8, 9], machine learning-based methods have been proposed for DDI extraction on the public corpora of the challenges. Both DDIExtraction 2011 and DDIExtraction 2013 are designed to extract drug-drug interactions from biomedical texts. DDIs without type information are labeled in the DDIExtraction 2011 corpus, while, in the DDIExtraction 2013 challenge, 


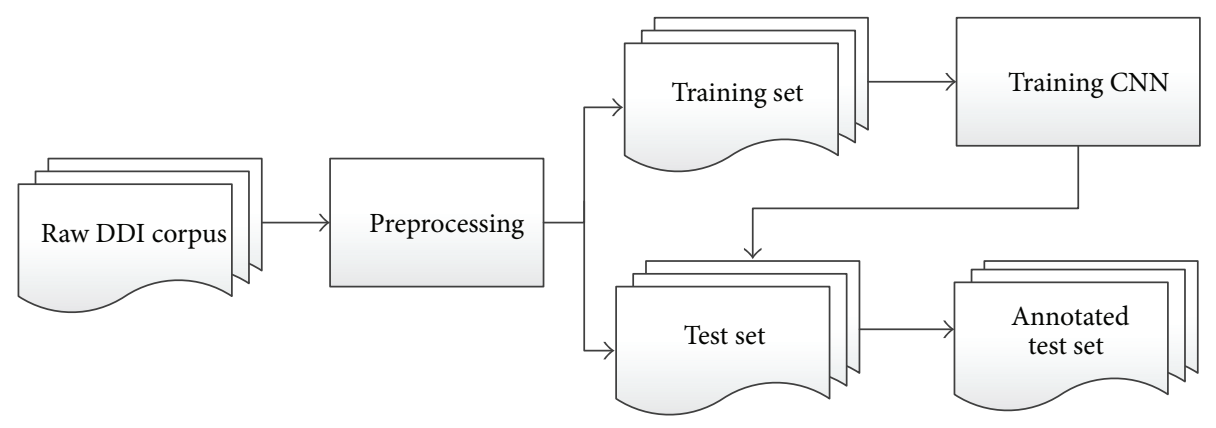

FIGURE 1: Overall workflow of the CNN-based method for DDI extraction.

DDIs are divided into four types, that is, "mechanism," "effect," "advice," and "int." The top performing systems on these corpora are based on support vector machines (SVM) with a large number of manually defined features [10-12]. For example, the best system of the 2013 DDIExtraction challenge [11] is based on SVM with a hybrid kernel using trigger words, dependency tree and parse tree features, and so forth. The subsequential best system [12] is based on linear SVM with rich features, including word, word pair, dependency graph, parse tree, and noun phrase-constrained coordination features. These systems have to suffer from fussy feature engineering. Most of features used in these systems are usually generated by existing NLP toolkits which are imperfect. Errors caused by the NLP toolkits inevitably propagate in the DDI extraction systems.

Convolutional neural networks $(\mathrm{CNN})$, a robust machine learning method proposed recently which almost does not need manually defined features, has exhibited great potential for many NLP tasks such as sentiment analysis [19], semantic parsing [20], and search query retrieval [21]. However, it has never been used for DDI extraction. In this paper, we deploy CNN for this task. Inputs of the CNN-based method are sentences in which drugs are annotated. The CNNbased method consists of four layers: a look-up table layer, a convolutional layer, a max pooling layer, and a softmax layer. Given a sentence with two drugs of interest, in the look-up table layer, each word is represented by word embeddings [22] and position embeddings [23], and then the sentence is represented by a matrix that concatenates word embeddings and position embeddings of its words in the order of their occurrence. In the convolutional layer, the matrix of the sentence is convolved with filters of different sizes, generating a group of feature vectors. The number of feature vectors is equal to that of filters and the size of each vector is determined by the context window considered. In the max pooling layer, the group of vectors is converted into a new vector by reducing each vector in the group into a feature. Finally, the vector obtained in the max pooling layer is fed to the fully connected softmax layer for classification. The word embeddings used in the look-up table layer are initialized by the "Order" algorithm [22] on the MEDLINE abstracts in 2013 [24], whereas the position embeddings are randomly initialized.

Evaluation on the 2013 DDIExtraction challenge corpus demonstrates that the CNN-based DDI extraction system achieves a precision, a recall, and an $F$-score of $75.72 \%$, $64.66 \%$, and $69.75 \%$, respectively. It outperforms the best performing system by $2.75 \%$ in $F$-score, indicating that $\mathrm{CNN}$ is a good choice for DDI extraction.

\section{Methods}

DDI extraction is recognized as a multiclass classification problem for all possible interacting pairs of drugs in the same sentence. Each pair of drugs is classified into one of the predefined types of DDIs or classified as a noninteracting pair. Given a sentence with $n$ drugs, a total of $C_{n, 2}=n(n-1) / 2$ DDI candidates need to be classified. Figure 1 illustrates the overall workflow of our CNN-based method for DDI extraction.

The preprocessing module first blinds drugs, tokenizes sentences, normalizes tokens, and filters out noninteracting pairs from DDI candidates. Then the CNN module is used for DDI extraction. In the training phase, DDI candidates that annotated in the training set are positive samples with different types, and the other candidates are negative samples. The task of training is to obtain a CNN model on these samples. In the test phase, all DDI candidates are classified into different types of DDIs or non-DDI.

2.1. Preprocessing. To ensure generalization of machine learning-based methods, we follow previous studies [12, 25] to blind drugs in a sentence in the following way: the two drugs of interest are replaced by "drugl" and "drug2" in the order of their occurrence, respectively, and all the other drugs are replaced by "drug0." For example, given a sentence with four drugs, "When ALFENTA is administered in combination with other CNS depressants such as barbiturates, or tranquilizers," where drugs are highlighted in italic, $C_{4,2}=6 \mathrm{DDI}$ candidates with context (called instances) are generated, as shown in Table 1.

After drug blinding, we use the Natural Language Toolkit (NLTK) [26] to tokenize sentences and convert all words to lowercase.

Among all DDI instances, there are a large number of negative instances (noninteracting drug pairs with context), which usually affect the performances of machine learningbased DDI extraction systems because of data imbalance problem [27, 28]. Therefore, filtering out negative instances as many as possible is very important for subsequent DDI 
TABLE 1: DDI candidates in a sentence after drug blinding.

\begin{tabular}{ll}
\hline Drug pair & DDI candidate with context after drug blinding (i.e., instance) \\
\hline (ALFENTA, CNS depressants) & When $d r u g 1$ is administered in combination with other $d r u g 2$ such as drug0 or drug0 \\
(ALFENTA, barbiturates) & When $d r u g 1$ is administered in combination with other drug0 such as drug2 or drug0 \\
(ALFENTA, tranquilizers) & When $d r u g 1$ is administered in combination with other drug0 such as drug0 or drug2 \\
(CNS depressants, barbiturates) & When drug0 is administered in combination with other $d r u g 1$ such as drug2 or drug0 \\
(CNS depressants, tranquilizers) & When drug0 is administered in combination with other drug1 such as drug0 or drug2 \\
(barbiturates, tranquilizers) & When drug0 is administered in combination with other drug0 such as drug1 or drug2 \\
\hline
\end{tabular}

extraction module. In this study, we define the following four criteria for negative instance filtering. An instance, denoted by "drug1, drug2," is a negative instance if

(1) the two drugs have the same name,

(2) one drug is an abbreviation or acronym of the other,

(3) the two drugs appear in the same coordinate structure that has more than two drugs as elements,

(4) one drug is a special case of the other.

Exact string matching is used to determine whether the first criterion is satisfied, and some simple rules are defined to determine whether any one of the other three criteria is satisfied such as "drug1 (drug2)," "drug1, drug2, and drug0," and "drug1 such as drug2." For example, the fourth and fifth instances in Table 1 are negative instances because of criterion 4 .

\subsection{Convolutional Neural Networks for Drug-Drug Interaction} Extraction. The CNN model proposed for DDI extraction in this study is a four-layer model (shown in Figure 2), which is a variant of the model for sentence classification in [19]. Besides word embeddings, position embeddings [23] are also integrated into the CNN model in [19] to encode relative distances between words and the two drugs of interest.

2.2.1. Look-Up Table. The CNN model takes DDI instances as input and generates their representation in look-up table layer. As required by $\mathrm{CNN}$, we set all instances to be of the same length by appending padding, denoted by "\#," to short instances. The maximal length of all instances is a proper choice of the same length, denoted by $n$. Given a DDI instance $S=w_{1} w_{2} w_{3} \cdots w_{n}$ with two drugs of interest ("drug1" and "drug2") at positions $p_{1}$ and $p_{2}$, a word $w_{i}$ is represented by $d_{w}$-dimensional word embeddings $\mathbf{e}_{w_{i}}$ and $2 d_{p}$-dimensional position embeddings $\left[\mathbf{e}_{d_{i 1}}{ }^{\mathrm{T}}, \mathbf{e}_{d_{i 2}}{ }^{\mathrm{T}}\right]^{\mathrm{T}}$ looked up from corresponding dictionaries. $\mathbf{C} \in \mathbb{R}^{d_{w} \times|V|}, \mathbf{D}_{1} \in$ $\mathbb{R}^{d_{p} \times(2 n-1)}$, and $\mathbf{D}_{2} \in \mathbb{R}^{d_{p} \times(2 n-1)}$, where $V$ is the vocabulary and $d_{i 1}=i-p_{1}$ and $d_{i 2}=i-p_{2}$ (ranging from $-n+$ 1 to $n-1)$ are, respectively, the relative distance between the word and the first drug and that between the word and the second drug. That is, $w_{i}$ is represented by $\mathbf{x}_{i}=$ $\left[\mathbf{e}_{w_{i}}{ }^{\mathrm{T}}, \mathbf{e}_{d_{i 1}}{ }^{\mathrm{T}}, \mathbf{e}_{d_{i 2}}{ }^{\mathrm{T}}\right]^{\mathrm{T}}$. Then the instance is represented by a matrix that concatenates the word embeddings and position embeddings of its words in the order of their occurrence, denoted by $\mathbf{x}=\left[\mathbf{x}_{1}, \mathbf{x}_{2}, \mathbf{x}_{3}, \ldots, \mathbf{x}_{n}\right]$ of size $\left(d_{w}+2 d_{p}\right) \times n$.
For the two types of embeddings, word embeddings can be initialized by employing unsupervised word embeddings algorithm on large-scale unannotated texts, whereas position embeddings only can be randomly initialized.

2.2.2. Convolution. The matrix of a DDI instance (i.e., $\mathbf{x}$ ) is fed to the convolutional layer to generate features by convolving $\mathbf{x}$ with filters of different sizes. Given a filter of size $k, \mathbf{t} \in \mathbb{R}^{\left(d_{w}+2 d_{p}\right) \times k}$, for example, the following feature $f_{i}$ can be generated by applying convolution operator to a context window of $k$ words:

$$
f_{i}=\tanh \left(\mathbf{t} \cdot \mathbf{x}_{i: i+k-1}+b\right) \text {, }
$$

where $\mathbf{x}_{i: i+k-1}$ denotes the matrix $\left[\mathbf{x}_{i}, \mathbf{x}_{i+1}, \mathbf{x}_{i+2}, \ldots, \mathbf{x}_{i+k-1}\right]$ (representation of words in the context window), $b \in \mathbb{R}$ is a bias, and tanh is the hyperbolic tangent function. When filter $\mathbf{t}$ is applied to all possible context windows of $k$ words (i.e., $i$ ranging from 1 to $n-k+1$ ), a feature vector $\mathbf{f}=$ $\left[f_{1}, f_{2}, f_{3}, \ldots, f_{n-k+1}\right]\left(\mathbf{f} \in \mathbb{R}^{n-k+1}\right)$ is generated. As there are various types of filters of different sizes, we can obtain a group of feature vectors. The number of feature vectors is equal to the number of filters.

2.2.3. Max Pooling. The max pooling layer extracts the most important feature from each feature vector to reduce the computational complexity of subsequent layers. Concretely, the feature of maximum value $\widehat{f}=\max \left\{f_{1}, f_{2}, f_{3}, \ldots\right.$, $\left.f_{n-k+1}\right\}$ is extracted to represent a feature vector $\mathbf{f}=$ $\left[f_{1}, f_{2}, f_{3}, \ldots, f_{n-k+1}\right]$. Correspondingly, if there are $l$ feature filters, the matrix of a DDI instance (i.e., $\mathbf{x}$ ) is converted into a new vector of length $l$, denoted by $\mathbf{z}=\left[\widehat{f}_{1}, \widehat{f}_{2}, \widehat{f}_{3}, \ldots, \widehat{f}_{l}\right]$, where $\widehat{f}_{i}$ is the feature extracted from the $i$ th feature vector.

2.2.4. Softmax Regression. To prevent neural networks from overfitting, we follow [19] to randomly drop out units (along with their connections) from the networks during training. The feature vector $\mathbf{z}$ obtained by max pooling is not directly fed to the fully connected softmax layer for classification. Firstly, we randomly set each element of $\mathbf{z}$ to zero with a probability $p$ (following the Bernoulli distribution) and obtain a new feature vector $\mathbf{z}_{d}$. Then the vector $\mathbf{z}_{d}$ is fed to the fully connected softmax layer. At test time, the feature vector $\mathbf{z}$ is directly fed to the softmax layer for classification without dropout. 


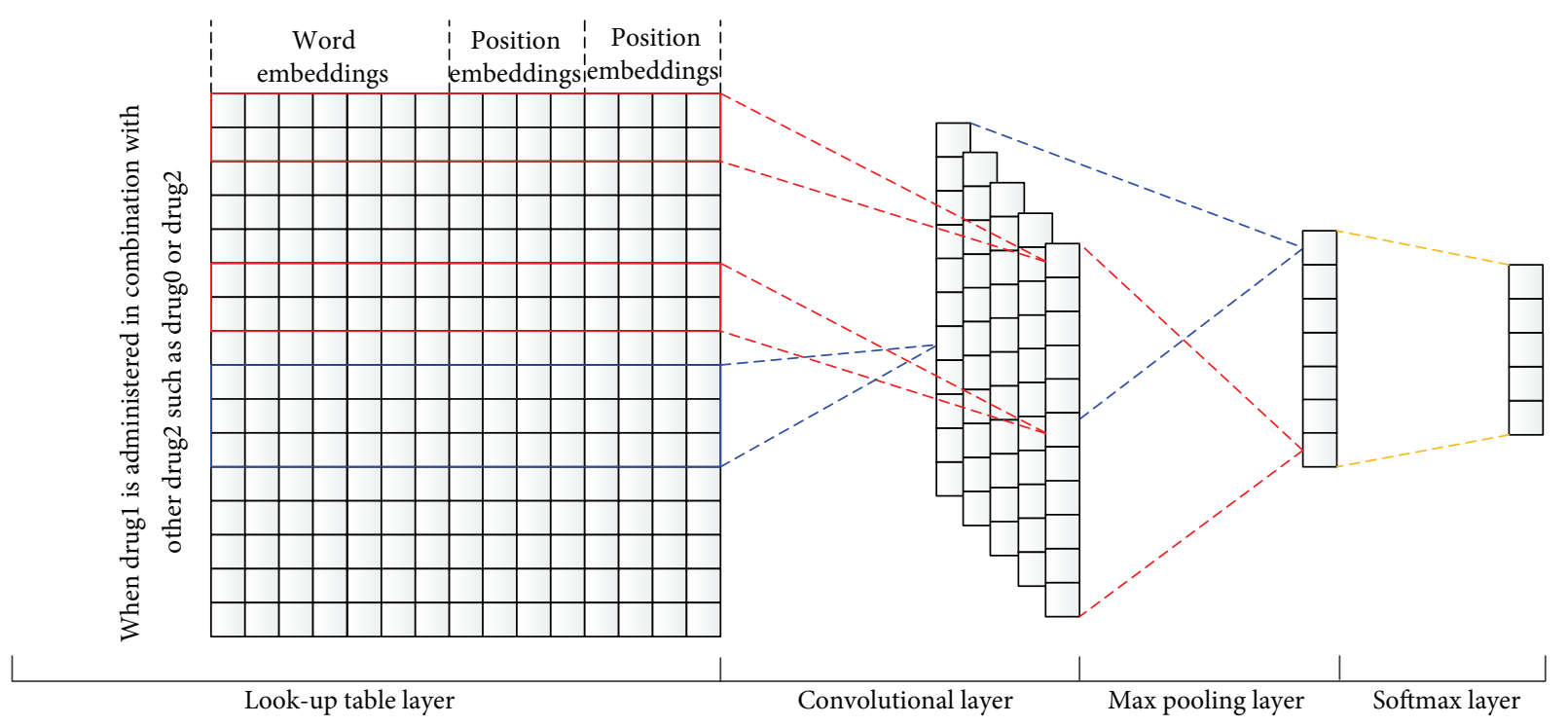

FIgURE 2: Architecture of the CNN model for DDI extraction.

2.2.5. Model Training. The following parameters of the CNN model need to be updated during training: the word embeddings matrix, the position embeddings matrixes, the filters, and the weight matrix of the softmax layer. We use stochastic gradient descent with shuffled minibatches and the AdaDelta update rule as [19] to learn the parameters. At each gradient descent step, we rescale the weight vectors of the softmax layer when their $l_{2}$-norms exceed a certain threshold.

\section{Experiments}

3.1. Dataset. The CNN-based DDI extraction system is developed and evaluated on the DDI corpus of the 2013 DDIExtraction challenge [29], which is composed of 730 DrugBank documents and 175 MEDLINE abstracts about DDIs. The corpus is split into two parts: a training set (572 DrugBank documents and 142 MEDLINE abstracts) for system development and a test set (158 DrugBank documents and 33 MEDLINE abstracts) for system evaluation (see Table 2). All drugs and pairs of drugs in each sentence are annotated. Among the pairs of drugs (totally 33508), 5000 interacting pairs (i.e., DDIs) are classified into the following four types: mechanism, effect, advice, and int. The definitions of the four types of DDIs are as follows.

(i) Mechanism. Mechanism is assigned when pharmacokinetic mechanism of a DDI is described.

(ii) Effect. Effect is assigned when effect of a DDI is described.

(iii) Advice. Advice is assigned when a recommendation or advice regarding a DDI is given.

(iv) Int. Int is assigned when the sentence simply states that a DDI occurs and does not provide any information about the DDI.
TABLE 2: Statistics of the DDI corpus of the 2013 DDIExtraction challenge.

\begin{tabular}{lcccc}
\hline & \multicolumn{2}{c}{ Training set } & \multicolumn{2}{c}{ Test set } \\
& DrugBank & MEDLINE & DrugBank & MEDLINE \\
\hline Documents & 572 & 142 & 158 & 33 \\
Pairs & 26005 & 1787 & 5265 & 451 \\
Positive DDIs & 3789 & 232 & 884 & 95 \\
Negative DDIs & 22216 & 1555 & 4381 & 356 \\
Mechanism & 1257 & 62 & 278 & 24 \\
Effect & 1535 & 152 & 298 & 62 \\
Advice & 818 & 8 & 214 & 7 \\
Int & 179 & 10 & 94 & 2 \\
\hline
\end{tabular}

3.2. Experimental Settings. As four types of DDIs are defined in the corpus of the 2013 DDIExtraction challenge, DDI instances need to be classified into five categories: mechanism, effect, advice, int, and noninteracting, corresponding to the output of the last layer of the CNN model.

The word embeddings matrix used in our experiments is initialized by an unsupervised word embeddings learning algorithm "Order" [22] on 17.3-gigabyte unannotated article abstracts extracted from MEDLINE released in 2013 [24]. We also adopt the NLTK to preprocess the abstracts, including splitting them into sentences, tokenizing the sentences, and converting all words to lowercase. Finally, we obtain 110 million sentences with 2.8 billion words from a vocabulary of size 1.99 million. Following previous works [19], we set the dimension of word embeddings to 300 and randomly initialized word embeddings of words not present in the vocabulary. For the position embeddings matrixes, we follow [23] to randomly initialize the position embeddings and determine the dimension of position embeddings heuristically (finally set to 10). 
The maximal length of the DDI instances is set to 150 , that is, the maximal length of sentences in the DDIExtraction 2013 corpus. Following [19], we used three kinds of filters for convolution; that is, $k$ is set to 3,4 , and 5 for filter $\mathbf{t} \in \mathbb{R}^{\left(d_{w}+2 d_{p}\right) \times k}$, and we used 200 filters of each kind at the convolutional layer. The dropout rate $(p), l_{2}$-norm threshold, and minibatch size are, respectively, set to $0.5,3$, and 50 , the same as [19]. Our CNN-based DDI extraction system will be released after the publication of this study.

To investigate the effect of different factors, we start with a baseline system without using position embeddings and negative instance filtering module and then add them gradually. We also compare our system with other stateof-the-art systems. The performances of all DDI extraction systems are measured by precision $(P)$, recall $(R)$, and $F$-score $(F)$, which are calculated by the evaluation tool provided by the 2013 DDIExtraction challenge organizers [30].

3.3. Experimental Results. The overall precision, recall, and $F$-score of our system are $75.72 \%, 64.66 \%$, and $69.75 \%$, as shown in Table 3, where the best performances are emphasized in bold. On the DrugBank subset, our system achieves an $F$-score of $71.52 \%$, which is higher than that on the MEDLINE subset by $19.40 \%$. Among four types of DDIs, our system performs best on advice instances and worst on int instances. The difference between the $F$-scores on these two types of DDIs achieves $31.37 \%$ (77.75\% versus $46.38 \%$ ).

Both position embeddings and negative instance filtering improve the overall performance of the CNN-based DDI extraction system. The improvements from them are $2.01 \%$ (67.01\% versus $65.00 \%)$ and $0.62 \%$ (65.62\% versus $65.00 \%)$ in $F$-score, respectively. When both of them are added to the baseline system, the CNN-based system is further improved by a total increase of $F$-score of $4.75 \%$ (69.75\% versus $65.00 \%)$. The system using both position embeddings and negative instance filtering shows much better performance than other systems on the DrugBank subset but worse performance than the system only using negative instance filtering by $3.05 \%$ on the MEDLINE subset. On all the four types of DDIs except int, the system using both position embeddings and negative instance filtering achieves better $F$ score than other systems. On int, the baseline system achieves best performance.

Compared with other state-of-the-art systems, including the best existing system and all participating systems of the 2013 DDIExtraction challenge (8 systems), our CNN-based system shows much better performance. It outperforms the current best system (Kim et al. [12]) by $2.75 \%$ and the best system of the 2013 DDIExtraction challenge (FBK-irst [11]) by $4.65 \%$ in $F$-score (see Table 4 ), mainly due to much higher precision. Top performing systems in Table 4 (e.g., Kim et al. [12], FBK-irst [11], and WBI [13]) are all based on SVM with a large number of manually defined features such as word, word pair, and dependency graph, as mentioned in Section 1.

\section{Discussion}

In this study, we propose a CNN-based system to extract DDIs in biomedical texts. To the best of our knowledge, it is the first time to use CNN for DDI extraction. As shown in Table 4, our CNN-based system outperforms all existing systems, most of which are based on SVM with various features such as syntactic feature $[11,12]$ and features derived from external lexical resources $[13,14]$. Compared with the state-of-the-art SVM-based systems, the advantage of our CNN-based system lies in that it does not use any manually defined features generated by existing NLP toolkits. The features used in the CNN-based system (i.e., word embeddings and position embeddings automatically learnt during training) may contain other useful information beyond the manually defined features. Moreover, they effectively avoid errors caused by existing NLP toolkits.

Position embedding improves the performance of our system on the DrugBank subset, but not on the MEDLINE subset. The main reason is that the position distribution of words in the DrugBank subset is more similar to that in the training set than the MEDLINE subset. To prove this point, we compare the average distance between two drugs of interest in the training set with that in the two test subsets and find that the difference between the average distance in the training set (18.06) and the average distance in the DrugBank subset (15.07) is much smaller than that between the training set and the MEDLINE subset (8.55).

The same as previous studies [11,27], negative instance filtering is beneficial to our system. The negative instance filtering module used in our system removes a large number of negative instances, but almost no positive instances. In the training set, 11206 out of 23771 negative instances are correctly filtered out. In the test set, 2698 out of 4737 negative instances are correctly filtered out, whereas only 7 out of 979 positive instances are wrongly filtered out. On the whole, more than $50 \%$ negative instances are correctly filtered out, but less than $0.2 \%$ positive instances are wrongly filtered out.

Our system shows much better performance on the DrugBank subset compared to the MEDLINE subset. There may be two reasons: (1) MEDLINE abstracts are usually written in scientific language. Long and complex sentences are commonly used in MEDLINE abstracts. In contrast, sentences in DrugBank are usually short and concise; (2) samples in the training set from MEDLINE are much less than DrugBank.

It is easy to understand that our system performs worst on int instances because of their proportionally small number among four types of DDI instances. The int instances only account for $4.7 \%(189 / 4021)$ in the training set. A possible direction for improvement is to take the imbalanced distributions of different types of instances into account like [31].

Although our system outperforms all other existing systems, there also are a large number of errors in our system (listed in Table 5, where the numbers on the two sides of plus signs are negative instances predicted by the CNN model and negative instance filtering module, resp.). Most of errors occur between positive instances and negative instances. 277 out of 979 positive instances are wrongly classified into negative instances (false negative instances). 134 negative instances are wrongly classified into positive instances (false positive instances). A small number of errors between four different types of DDIs (69 out of 979) occur in our system. 


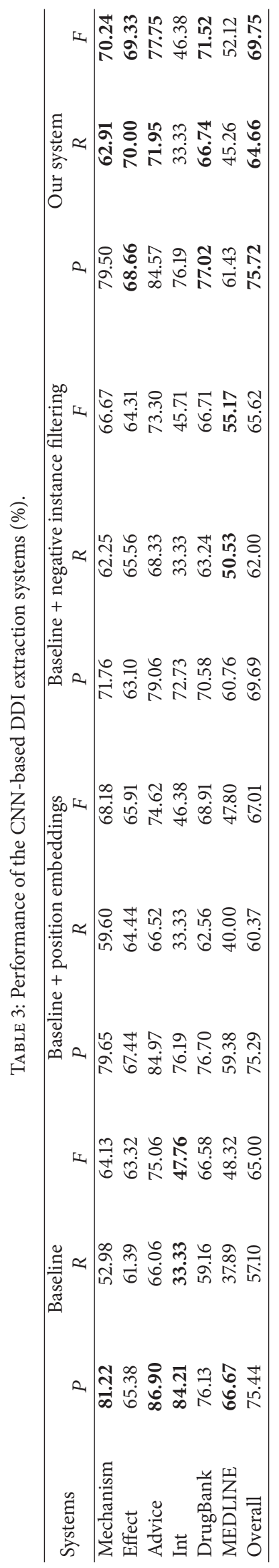


TABLE 4: Comparison between our CNN-based system and other state-of-the-art systems (\%).

\begin{tabular}{|c|c|c|c|c|c|c|c|c|c|}
\hline \multirow{2}{*}{ Systems } & \multicolumn{3}{|c|}{ DrugBank } & \multicolumn{3}{|c|}{ MEDLINE } & \multicolumn{3}{|c|}{ Overall } \\
\hline & $P$ & $R$ & $F$ & $P$ & $R$ & $F$ & $P$ & $R$ & $F$ \\
\hline Our system & 77.02 & 66.74 & 71.52 & 61.43 & 45.26 & 52.12 & 75.72 & 64.66 & 69.75 \\
\hline Kim et al. [12] & - & - & 69.80 & - & - & 38.20 & - & - & 67.00 \\
\hline FBK-irst [11] & 66.70 & 68.60 & 67.60 & 41.90 & 37.90 & 39.80 & 64.60 & 65.60 & 65.10 \\
\hline WBI [13] & 65.70 & 60.90 & 63.20 & 45.30 & 30.50 & 36.50 & 64.20 & 57.90 & 60.90 \\
\hline UTurku [14] & 73.80 & 53.50 & 62.00 & 59.30 & 16.80 & 26.20 & 73.20 & 49.90 & 59.40 \\
\hline NIL_UCM [15] & 56.60 & 57.90 & 57.30 & 35.70 & 15.80 & 21.90 & 55.70 & 53.80 & 54.80 \\
\hline UC3M & 51.80 & 59.80 & 55.50 & 26.50 & 28.40 & 27.40 & 49.50 & 56.80 & 52.90 \\
\hline UWM-TRIADS [16] & 45.20 & 52.40 & 48.50 & 31.20 & 32.60 & 31.90 & 43.90 & 50.50 & 47.00 \\
\hline SCAI [17] & 54.60 & 40.40 & 46.40 & 62.50 & 31.60 & 42.00 & 55.10 & 39.50 & 46.00 \\
\hline UColorado_SOM [18] & 28.80 & 44.10 & 34.90 & 17.30 & 41.10 & 24.40 & 27.20 & 43.80 & 33.60 \\
\hline
\end{tabular}

TABLE 5: Prediction Results of our CNN-based DDI extraction system.

\begin{tabular}{lcccccc}
\hline \multicolumn{2}{c}{ Gold standard annotation } & & \multicolumn{3}{c}{ Prediction results } \\
Type & Total number & Mechanism & Effect & Advice & Int & Negative \\
\hline Mechanism & 302 & 190 & 8 & 252 & 3 & 0 \\
Effect & 360 & 6 & 1 & 159 & 1 & $96+1$ \\
Advice & 221 & 2 & 39 & 0 & 2 & 32 \\
Int & 96 & 0 & 67 & 19 & $75+2$ \\
Negative & 4737 & 41 & & & 7 \\
\end{tabular}

Among these errors, 39 int instances are wrongly classified into effect instances, accounting for $56.52 \%$. Reducing errors between positive instances and negative instances will greatly improve the CNN-based DDI extraction system, which is part of our future work.

\section{Conclusions}

In this paper, we propose a CNN-based method for DDI extraction. Word embeddings and position embeddings, which capture the semantic information of words and relative distances between words and two drugs of interest, respectively, are used to represent DDI instances. Experiments on the 2013 DDIExtraction challenge corpus demonstrate that the proposed CNN-based method outperforms other stateof-the-art methods on DDI extraction. It is the first time to apply CNN to DDI extraction. In our CNN-based method, not only word embeddings but also position embeddings are considered. Both of them do not rely on any existing NLP toolkits.

\section{Conflict of Interests}

The authors declare that there is no conflict of interests regarding the publication of this paper.

\section{Authors' Contribution}

The work presented here was a collaboration of all the authors. All authors contributed to designing the method and experiments. Shengyu Liu performed the experiments.
All authors analyzed the data and interpreted the results. Shengyu Liu drafted the paper and all authors revised it. All authors have read and approved the paper. Shengyu Liu and Buzhou Tang contributed equally to this work.

\section{Acknowledgments}

This paper is supported in part by the following grants: National 863 Program of China (2015AA015405), NSFCs (National Natural Science Foundations of China) (61573118, 61402128, 61473101, 61472428, 61173075, and 61272383), Science and Technology Funds of Guizhou Province (no. [2013]2335), and Strategic Emerging Industry Development Special Funds of Shenzhen (JCYJ20140508161040764, JCYJ20140417172417105, and JCYJ20140627163809422).

\section{References}

[1] I. Segura-Bedmar, P. Martínez, and M. Herrero-Zazo, "Lessons learnt from the DDIExtraction-2013 shared task," Journal of Biomedical Informatics, vol. 51, pp. 152-164, 2014.

[2] V. Law, C. Knox, Y. Djoumbou et al., "DrugBank 4.0: shedding new light on drug metabolism," Nucleic Acids Research, vol. 42, no. 1, pp. D1091-D1097, 2014.

[3] J. K. Aronson, "Communicating information about drug interactions," British Journal of Clinical Pharmacology, vol. 63, no. 6, pp. 637-639, 2007.

[4] I. Segura-Bedmar, P. Martínez, and C. de Pablo-Sánchez, "A linguistic rule-based approach to extract drug-drug interactions from pharmacological documents," BMC Bioinformatics, vol. 12, supplement 2, no. 1, 2011. 
[5] I. Segura-Bedmar, P. Martínez, and C. de Pablo-Sánchez, "Using a shallow linguistic kernel for drug-drug interaction extraction," Journal of Biomedical Informatics, vol. 44, no. 5, pp. 789-804, 2011.

[6] Q. Bui, P. M. Sloot, E. M. van Mulligen, and J. A. Kors, "A novel feature-based approach to extract drug-drug interactions from biomedical text," Bioinformatics, vol. 30, no. 23, pp. 3365-3371, 2014.

[7] D. Zhou, D. Zhong, and Y. He, "Biomedical relation extraction: from binary to complex," Computational and Mathematical Methods in Medicine, vol. 2014, Article ID 298473, 18 pages, 2014.

[8] I. Segura-Bedmar, P. Martínez, and D. Sánchez-Cisneros, “The 1st DDIExtraction-2011 challenge task: extraction of drugdrug interactions from biomedical texts ", in Proceedings of the 1st Challenge Task on Drug-Drug Interaction Extraction (DDIExtraction '11), pp. 1-9, Huelva, Spain, September 2011.

[9] I. Segura-Bedmar, P. Martínez, and M. Herrero-Zazo, "SemEval-2013 task 9: extraction of drug-drug interactions from biomedical texts (DDIExtraction 2013)," in Proceedings of the 7th International Workshop on Semantic Evaluation (SemEval '13), pp. 341-350, Atlanta, Ga, USA, June 2013.

[10] P. Thomas, M. Neves, I. Solt, D. Tikk, and U. Leser, "Relation extraction for drug-drug interactions using ensemble learning," in Proceedings of the 1st Challenge Task on Drug-Drug Interaction Extraction, pp. 11-18, Huelva, Spain, September 2011.

[11] M. Chowdhury and A. Lavelli, "FBK-irst: a multi-phase kernel based approach for drug-drug interaction detection and classification that exploits linguistic information," in Proceedings of the 7th International Workshop on Semantic Evaluation (SemEval '13), pp. 351-355, Atlanta, Ga, USA, June 2013.

[12] S. Kim, H. Liu, L. Yeganova, and W. J. Wilbur, "Extracting drugdrug interactions from literature using a rich feature-based linear kernel approach," Journal of Biomedical Informatics, vol. 55, pp. 23-30, 2015.

[13] P. Thomas, M. Neves, T. Rocktäschel, and U. Leser, "WBIDDI: drug-drug interaction extraction using majority voting," in Proceedings of the 7th International Workshop on Semantic Evaluation, pp. 628-635, Atlanta, Ga, USA, June 2013.

[14] J. Björne, S. Kaewphan, and T. Salakoski, "UTurku: drug named entity recognition and drug-drug interaction extraction using SVM classification and domain knowledge," in Proceedings of the 7th International Workshop on Semantic Evaluation (SemEval '13), pp. 651-659, Atlanta, Ga, USA, June 2013.

[15] B. Bokharaeian and A. Díaz, "NIL-UCM: extracting drug-drug interactions from text through combination of sequence and tree kernels," in Proceedings of the 7th International Workshop on Semantic Evaluation (SemEval '13), pp. 644-650, Atlanta, Ga, USA, June 2013.

[16] M. Rastegar-Mojarad, R. Boyce, and R. Prasad, "UWMTRIADS: classifying drug-drug interactions with two-stage SVM and post-processing," in Proceedings of the 7th International Workshop on Semantic Evaluation, pp. 667-674, Atlanta, Ga, USA, June 2013.

[17] T. Bobić, J. Fluck, and M. Hofmann-Apitius, "SCAI: extracting drug-drug interactions using a rich feature vector," in Proceedings of the 7th International Workshop on Semantic Evaluation, pp. 675-683, Atlanta, Ga, USA, June 2013.

[18] N. Hailu, L. Hunter, and K. Cohen, "UColorado_SOM: extraction of drug-drug interactions from biomedical text using knowledge-rich and knowledge-poor features," in Proceedings of the 7th International Workshop on Semantic Evaluation (SemEval '13), pp. 684-688, Atlanta, Ga, USA, June 2013.

[19] Y. Kim, "Convolutional neural networks for sentence classification," in Proceedings of the Conference on Empirical Methods in Natural Language Processing (EMNLP '14), pp. 1746-1751, Doha, Qatar, October 2014.

[20] W. Yih, X. He, and C. Meek, "Semantic parsing for singlerelation question answering," in Proceedings of the 52nd Annual Meeting of the Association for Computational Linguistics (ACL '14), pp. 643-648, Baltimore, Md, USA, June 2014.

[21] Y. Shen, X. He, J. Gao, L. Deng, and G. Mesnil, "Learning semantic representations using convolutional neural networks for web search," in Proceedings of the 23rd International Conference on World Wide Web (WWW '14), pp. 373-374, Seoul, Republic of Korea, April 2014.

[22] S. Lai, K. Liu, L. Xu, and J. Zhao, "How to generate a good wordembedding?” http://arxiv.org/abs/1507.05523.

[23] D. Zeng, K. Liu, S. Lai, G. Zhou, and J. Zhao, "Relation classification via convolutional deep neural network," in Proceedings of the 25th International Conference on Computational Linguistics, pp. 2335-2344, Dublin, Ireland, August 2014.

[24] MEDLINE, http://www.nlm.nih.gov/databases/journal.html.

[25] L. He, Z. Yang, Z. Zhao, H. Lin, and Y. Li, "Extracting drugdrug interaction from the biomedical literature using a stacked generalization-based approach," PLoS ONE, vol. 8, no. 6, Article ID e65814, 2013.

[26] NLTK, http://www.nltk.org/.

[27] M. Chowdhury and A. Lavelli, "Impact of less skewed distributions on efficiency and effectiveness of biomedical relation extraction," in Proceedings of the 24th International Conference on Computational Linguistics (COLING '12), pp. 205-216, Bombay, India, December 2012.

[28] A. Sun, R. Grishman, and S. Sekine, "Semi-supervised relation extraction with large-scale word clustering," in Proceedings of the 49th Annual Meeting of the Association for Computational Linguistics, pp. 521-529, June 2011.

[29] M. Herrero-Zazo, I. Segura-Bedmar, P. Martínez, and T. Declerck, "The DDI corpus: an annotated corpus with pharmacological substances and drug-drug interactions," Journal of Biomedical Informatics, vol. 46, no. 5, pp. 914-920, 2013.

[30] Evaluation scripts of the 2013 DDIExtraction challenge, https:// www.cs.york.ac.uk/semeval-task9/index.php\%3Fid=evaluation .html.

[31] S. Wang and X. Yao, "Multiclass imbalance problems: analysis and potential solutions," IEEE Transactions on Systems, Man, and Cybernetics, Part B: Cybernetics, vol. 42, no. 4, pp. 1119-1130, 2012. 


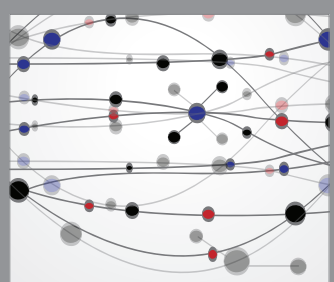

The Scientific World Journal
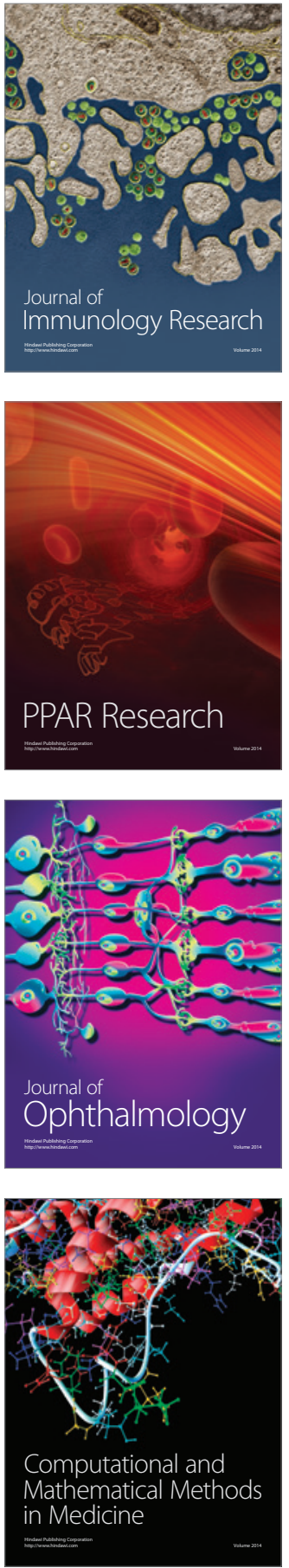

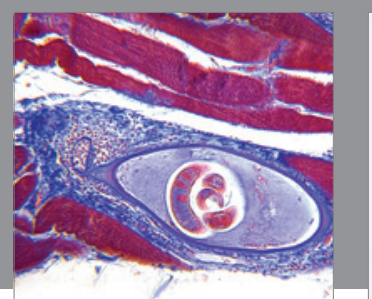

Gastroenterology Research and Practice

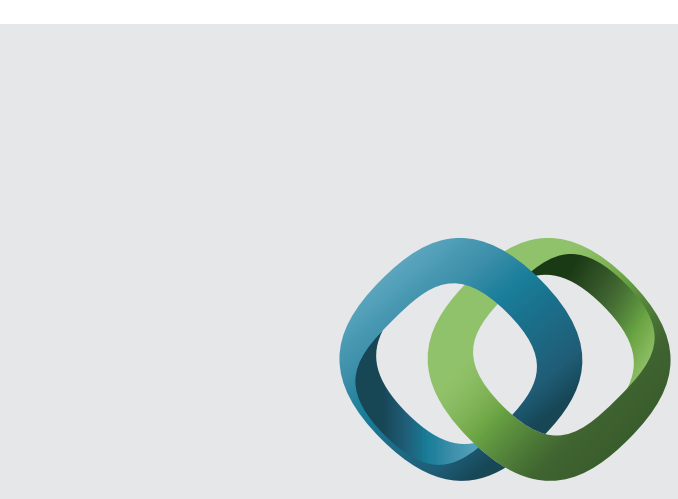

\section{Hindawi}

Submit your manuscripts at

http://www.hindawi.com
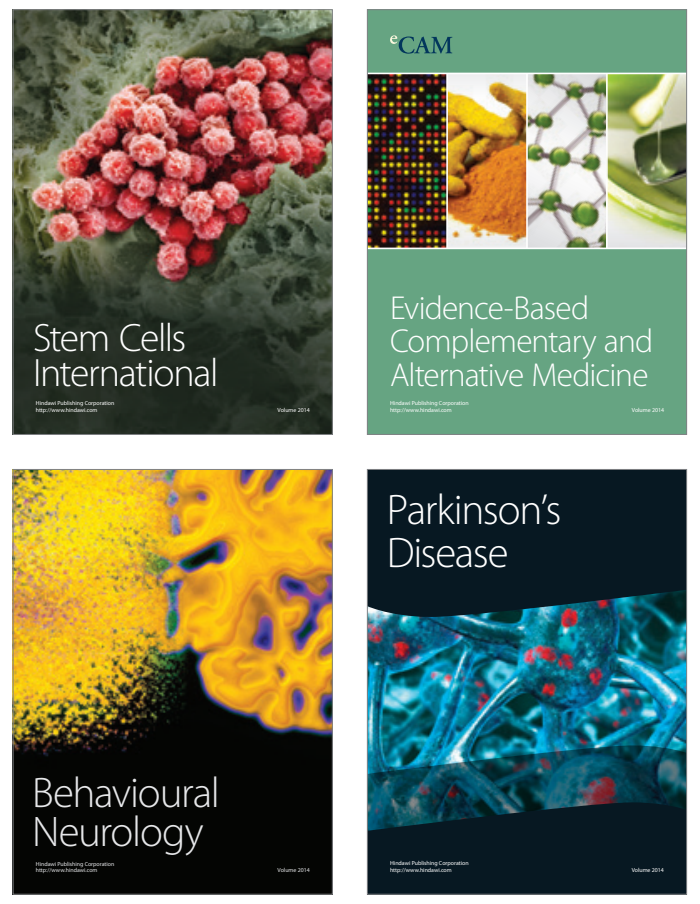
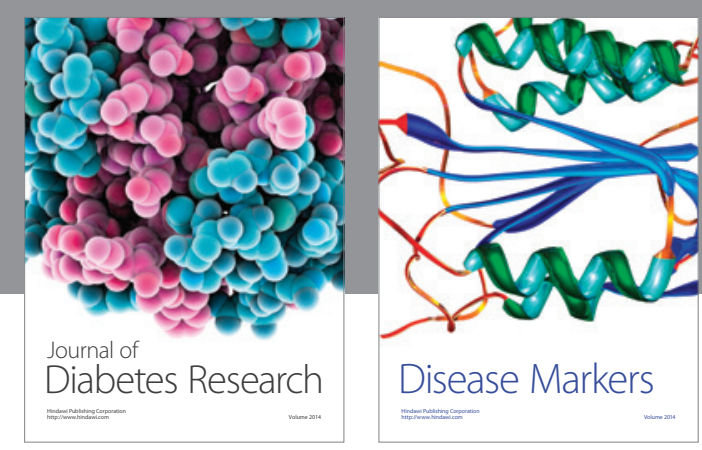

Disease Markers
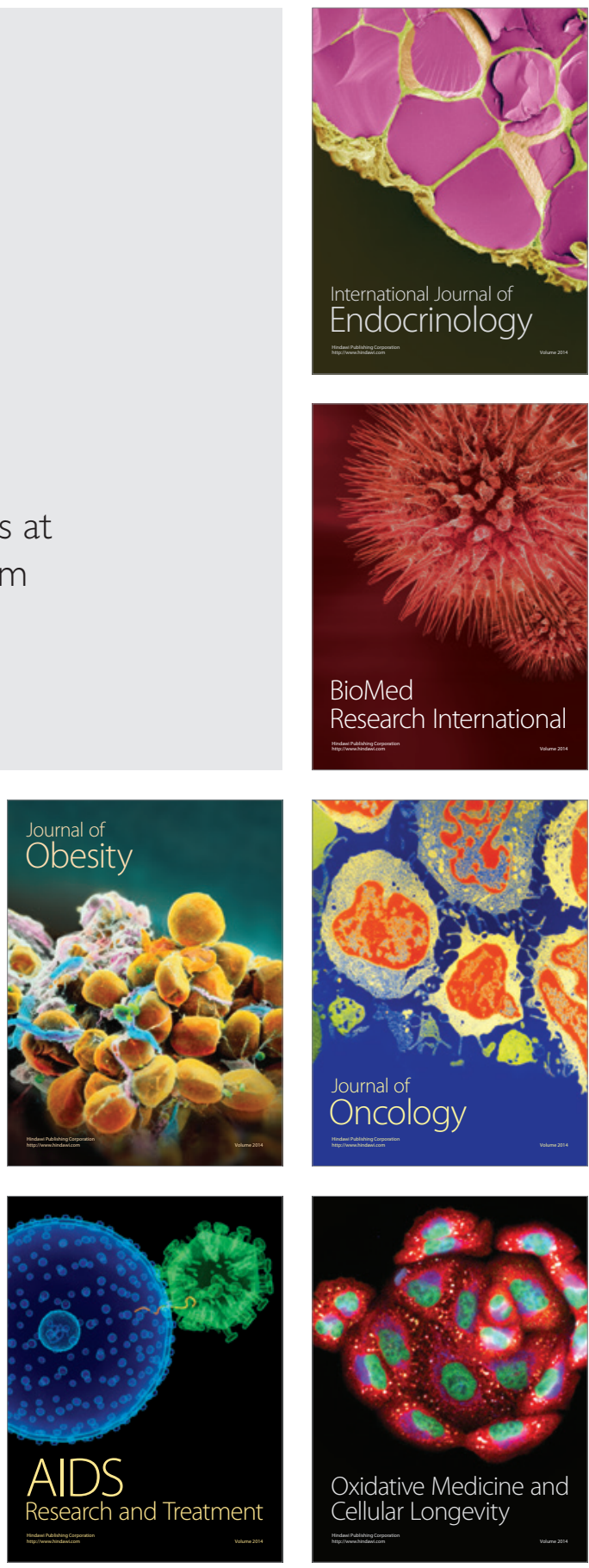